University of Nebraska - Lincoln

DigitalCommons@University of Nebraska - Lincoln

U.S. Environmental Protection Agency Papers

U.S. Environmental Protection Agency

1997

\title{
Quantitation Reference Compounds and VOC Recoveries from Soils By Purge-and-Trap GC/MS
}

Brian Schumacher

U.S. EPA

Steven Ward

Harry Reid Center for Environmental Studies, University of NevadasLas Vegas, 4505 Maryland Park, Las

Vegas, Nevada

Follow this and additional works at: https://digitalcommons.unl.edu/usepapapers

Part of the Civil and Environmental Engineering Commons

Schumacher, Brian and Ward, Steven, "Quantitation Reference Compounds and VOC Recoveries from Soils By Purge-and-Trap GC/MS" (1997). U.S. Environmental Protection Agency Papers. 66.

https://digitalcommons.unl.edu/usepapapers/66

This Article is brought to you for free and open access by the U.S. Environmental Protection Agency at DigitalCommons@University of Nebraska - Lincoln. It has been accepted for inclusion in U.S. Environmental Protection Agency Papers by an authorized administrator of DigitalCommons@University of Nebraska - Lincoln. 


\section{Quantitation Reference Compounds and VOC Recoveries from Soils By Purge-and-Trap GC/MS}

\begin{abstract}
BRIAN A. SCHUMACHER
U.S. Environmental Protection Agency, National Exposure Research Laboratory, Characterization Research DivisionLas Vegas, Analytical Sciences Branch, P.O. Box 93478, Las Vegas, Nevada 89193
\end{abstract}

STEVEN E. WARD*

Harry Reid Center for Environmental Studies, University of Nevada-Las Vegas, 4505 Maryland Park, Las Vegas, Nevada 89154

Unquantified volatile organic compound (VOC) losses occur in every phase of VOC determination including sample collection, transport, storage, preparation, and analysis. Current quality assurance/quality control measures, such as surrogate spikes and internal standards, do not account for losses that occur during sample handling nor do they account for soil matrix effects. An alternate approach, the quantitation reference compound (QRC) approach, is presented that involves direction injection of the QRCs onto the soil matrix to account for any matrix effects and losses during subsequent processing steps prior to sample analysis. Final VOC quantitation is based on the QRC instead of the internal standard, which is used stric tly to monitor instrument performance. Quantifying VOCs using the QRC approach on three spiked performance evaluation soils and one unprocessed spiked field soil resulted in target compound relative recoveries of $93-105 \%$. In contrast, using the current quantitation approach, target compound recoveries were only $2-27 \%$. Precision betw een the two approaches was equivalent (on an absolute basis) with relative standard deviations of $2-15 \%$ using the QRC approach.

\section{Introduction}

Volatile organic compounds (VOCs) present analytical problems different from those encountered for other organic contaminants, such as pesticides and polychlorinated biphenyls. Thesedifficulties arisedue to the capability of VOCs to readily change physical states with an affinity for the vapor phase. As a result of this affinity, VOC losses from samples occur through numerous pathways. In the field, losses may occur during sample transfer from the sampling device to collection vessel, during subsampling, during addition of preservants, or as a result of sample disaggregation $(1,2)$ or poor bottlesealing $(3,4)$. During sampletransport and storage prior to analysis, VOCs may be lost through biodegradation and abiotic degradation (5-10) or may becomeless purgeable by diffusion into intra-aggregate micropores (11). Lastly, losses may occur at thelaboratory when samples are decapped to be attached to the purge unit, during subsampling, and from punctured seals on vials $(12,13)$. Lossesin thelaboratory may befurther exacerbated by incomplete desorption during

* Corresponding author present address: DynCorp Inc., $300 \mathrm{~N}$. Lee St., Suite 500, Alexandria, VA 22314; telephone: (703)519-1320; e-mail: sward@dyniet.com. sample purging if a solid matrix, such as soil, is present (1416). Losses from these sources have been shown to be up to 3 orders of magnitude from known VOC concentrations (5, 17) and may account for the large number of non-detects associated with VOC data.

In an attempt to control and quantify VOC losses, quality assurance (QA) and quality control (QC) procedures are implemented at variousstages in the processing stream. These measures include using blanks (equipment, field, method, reagent, and trip), initial and ongoing calibration checks, sample replication, performance evaluation samples, matrix spikes, surrogate spikes, and the use of internal standards. Additionally, if gas chromatography/ mass spectrometry (GC/ $\mathrm{MS}$ ) is used, then GC/MS tuning and mass calibration are importantQA/QC tools. TheseQA/QC measures and samples are intended to control contamination from sources outside the sample, assess method performance, ensure proper instrument operation, and ultimately result in accurate, precise, and unbiased VOC quantification.

Internal standards are pure organic compounds that are similar in analytical behavior and not affected by the sample matrix (18). Internal standards are used in the initial instrument calibration, in the determination of response factors (RFs) for VOC quantitation, and to verify instrument operation and calibration on a daily basis. Similarly, surrogate spikes are pure organic compounds that are similar to the analytes of interest in chemical composition, extraction, and chromatography, but which are not normally found in environmental samples $(18,19)$. Surrogate spikes are added to every sample (including QA/QC samples) and used to examine the overall efficiency of the method from sample preparation through extraction and analysis. Generally, both the surrogate spike and internal standards are added to the sample just prior to purging $(18,19)$.

While the current use of internal standards and surrogate spikes provides useful information about instrument operation and can be used for VOC quantitation, concern has been expressed about their effectiveness in the analysis of VOCcontaminated soils. The concern arises because surrogate spikes are supposed to monitor the overall system efficiency including the efficiency of extraction from the matrix. However, since surrogate spikes are added to the sample just prior to purging, little time to react with the soil matrix is allowed, and the assessment of extraction efficiency is thus limited. In contrast, any VOCs in the soil sample have established a partitioning equilibrium in the container between the timethey aresealed and analyzed. The presence of soil sorption sites has been shown to reduce the extraction efficiency of purge-and-trap techniques (20-22). Thus, surrogate spikes placed in the extraction water do not fully represent the extraction process occurring between soil and water.

An additional concern arises since VOCs are quantified based on internal standard-generated RFs. Internal standards arepresent only in an aqueous media (i.e., generally, methanol for standard preparation and water for extraction) and by definition should not beaffected by the matrix (18). However, since VOCs in soil react differently than in water, the use of aqueous-based quantitation standards is questionable and the influence of the soil matrix is neglected. Further, the internal standard may come in contact and subsequently interact with the sample prior to purge initiation, thereby reducing its effectiveness for monitoring instrument performance.

To allow for more accurate and precise quantitation of soil VOCs regardless of losses during transport, subsampling, storage, and analysis, an alternate use for the surrogate spike 


\begin{tabular}{|c|c|c|c|c|}
\hline soil type & sand $(\%)$ & silt (\%) & clay (\%) & TOC ${ }^{a}$ \\
\hline $\begin{array}{l}\text { PE C horizon } \\
\text { PE } B^{b} \text { horizon } \\
\text { PE Bw horizon } \\
\text { Nebraska Ap horizon }\end{array}$ & $\begin{array}{r}95 \\
47 \\
26 \\
7\end{array}$ & $\begin{array}{r}4 \\
49 \\
65 \\
60\end{array}$ & $\begin{array}{r}1 \\
4 \\
9 \\
33\end{array}$ & $\begin{array}{l}0.1 \\
1.0 \\
3.0 \\
2.1\end{array}$ \\
\hline
\end{tabular}

and internal standard is proposed. In theproposed approach, the same compounds commonly used as internal standards and surrogate spikes will be used. However, their useas QA QC monitors and in VOC quantitation as well as their point of entry into the sampling and analytical stream will be different.

\section{Experimental Section}

Soils. Threehomogeneous performance evaluation (PE) soils, created for theEPA's National Acidic Preci pitation Assessment Program, were used as base materials for this project. The soils were oven-dried at $105^{\circ} \mathrm{C}$ overnight and analyzed for total organic carbon and particle-size distribution following Method 9060 (19) and the sieve and pipette method of Gee and Bauder (23), respectively (Table 1). Additionally, a fieldmoist Ap horizon collected from northern Nebraska was used to examine the effectiveness of the proposed approach on a heterogeneous (i.e., no sieving, drying, or homogenization) "real world" soil (Table 1). Prior to spiking the soils with VOCs, each soil was analyzed by purge-and-trap (P\&T) GC/ MS to ensure no initial VOC contamination.

Standards. Separate standard solutions were prepared from EPA traceablestock solutions, for theinternal, surrogate, and target compounds in methanol at concentrations of 0.25 $\mu \mathrm{g} / \mu \mathrm{L}$. The target compounds in this study were 1,1dichloroethane(1,1-DCA), 1,1,1-trichloroethane (1,1,1-TCA), benzene (Bz), trichloroethylene (TCE), bromodichloromethane $\left(\mathrm{BrCl}_{2} \mathrm{Me}\right)$, perchloroethylene (PCE), ethylbenzene (EBz), bromoform (Brmfrm), and 1,1,2,2-tetrachloroethane (1,1,2,2TTCA). The study surrogate standards were 1,2-dichloroethane- $d_{4}\left(1,2-D C A-d_{4}\right)$, toluene- $d_{8}\left(\right.$ Tol- $\left.d_{8}\right)$, and p-bromofluorobenzene (BFB). The study internal standards were chlorobenzene- $d_{5}\left(C I B z-d_{5}\right)$, bromochloromethane (BrCIMe), and 1,4-difluorobenzene (1,4-diFBz).

Sample Preparation. Twenty-four VOC-contaminated soils, divided into three sets of eight, were prepared for each PE soil by placing $5 \mathrm{~g}$ of soil into a $40-\mathrm{mL}$ VOA vial and adding a $100 \mu \mathrm{g} / \mathrm{kg}$ spike containing each target compound. All samples from each PE soil also had the surrogatespikes added at a $100 \mu \mathrm{g} / \mathrm{kg}$ concentration during target compound spiking. The vials were immediately capped with a screw top containing a Teflon-faced silicone septum. Samples were tumbled end over end for $120 \mathrm{~s}$ and stored at $4{ }^{\circ} \mathrm{C}$ for 3 days with the exception of the third batch of $C$ horizon samples that were stored for only 2 days.

Similar to the process used in preparation of the PE soil batches, 24 5-g Ap horizon samples, divided into three sets of eight, were spiked with target compounds and surrogate spikes such that a final concentration of $100 \mu \mathrm{g} / \mathrm{kg}$ was achieved. The vials were immediately capped with a screw top containing a Teflon-faced silicone septa. Samples were tumbled end over end for $120 \mathrm{~s}$ and stored at $4{ }^{\circ} \mathrm{C}$ for 3 days. A second batch of 24 samples, divided into three sets of eight, were spiked with target compounds, capped, and stored for 3 days at $4^{\circ} \mathrm{C}$ as before. Surrogate compounds were added to each set of eight samples by inversion of the VOA vial containing the soil and injecting surrogate compounds directly into the soil as it lay against the septa. These samples were not tumbled to mix the soil and surrogate spike. The first set of eight samples was analyzed $3 \mathrm{~h}$ after the addition of thesurrogatecompounds whiletheremaining two batches were analyzed 2 and 3 days after surrogate spike addition, respectively. This process resulted in theanalysis of samples with target compound holding times of 3, 5, and 6 days, respectively.

Instrumentation. Purging was performed on a Tekmar LSC-2000 apparatus, and separation and detection was performed using a Spectra-Physics GC/MS with Saturn software following Methods 5030 and 8260 (23). Instrument calibration was performed over the range of $10-100 \mu \mathrm{g} / \mathrm{kg}$ and checked on a continuing basis by direct injection of internal standards at a concentration of $50 \mu \mathrm{g} / \mathrm{kg}$ prior to sampledesorption and comparing its responseto theresponse from the initial calibration. A $60 \mathrm{~m}, 0.32 \mathrm{~mm}$ i.d., DB-624 column was used to allow for on-column injection. Conditions and settings used for these instruments are given in Table 2 .

\section{Results and Discussion}

Prior to discussion of the proposed approach, it is necessary to define several terms such that, when the current and proposed approach are discussed, confusion is avoided. The current approach to VOC quantitation is presented in Methods 5030 and $8260-P \& T$ GC/MS (23). The use of the terms surrogatespikes and internal standards are identical to those commonly used in these and other P\&T GC/MS methods. The proposed approach, to be referred to as the quantitation reference compound (QRC) approach, follows the same analytical procedures as specified in Methods 5030 and 8260 (i.e., the current approach) except for the useand introduction points of the surrogate spikes (to be known as the QRCs in this approach) and internal standards. The QRCs are the same compounds as the current method surrogate spikes except that they are spiked directly onto the soil during target compound spiking. TheQRCs will be used in the quantitation of VOCs instead of the internal standards (to be discussed). The function of the internal standards as monitors of the GC/MS system are the same between the two methods with the only difference being the introduction point in the QRC approach.

Current Method. In the currentmethod, surrogatespikes and internal standards are added to the sample in $5 \mathrm{~mL}$ of reagent water, and the sample is then purged. Surrogate spikes provide a QA/QC check and must meet acceptance criteria for the sample batch to be considered acceptable. Internal standards are paired with specific target compounds (based on similarities in analytical behavior and retention time) and used in the determination of the RFs from the initial calibration curve for each target compound as follows:

$$
\mathrm{RF}_{\mathrm{TC}}=\left(\mathrm{A}_{\mathrm{TC}} \mathrm{C}_{\mathrm{IS}}\right) /\left(\mathrm{A}_{\mathrm{IS}} \mathrm{C}_{\mathrm{TC}}\right)
$$

where $\mathrm{RF}_{\mathrm{TC}}$ is the response factor for target compound, $\mathrm{A}_{\mathrm{TC}}$ is the area of the characteristic ion for the target compound, $A_{I S}$ is the area of the characteristic ion of the internal standard, $\mathrm{C}_{\mathrm{TC}}$ is the concentration of target compound (ng), and $\mathrm{C}_{\mathrm{IS}}$ is the concentration of internal standard (ng). Final concentrations of soil VOCs are calculated as follows:

$$
\text { concentration }(\mu \mathrm{g} / \mathrm{kg})=\left(\mathrm{A}_{\mathrm{TC}} \mathrm{C}_{\mathrm{IS}}\right) /\left(\mathrm{A}_{\mathrm{IS}} \mathrm{RF}_{\mathrm{TC}} \mathrm{W}_{\mathrm{S}}\right)
$$

where $A_{T C}, C_{I S}$, and $A_{I S}$ are the same as above, $R_{T C}$ is the mean relative response factor for the target compound, and $\mathrm{W}_{\mathrm{S}}$ is the weight of sample $(\mathrm{g})$.

QRC Approach. In the QRC approach, QRCs are added directly to the sample as soon as possible to allow maximum time for soil/QRC interaction. In a field study, QRCs should beadded to the samples immediately after sample collection. If this is not possible, QRCs should be added immediately upon receipt at the analytical laboratory. In this study, QRCs were added at the time of initial soil spiking with the target 


\begin{tabular}{ll}
\multicolumn{2}{c}{ purge-and-trap settings } \\
\hline purge flow (He) & $40 \mathrm{~mL} / \mathrm{min}$ \\
sample purge temp & $40^{\circ} \mathrm{C}$ \\
purge time & $11 \mathrm{~min}$ \\
desorb temp & $180^{\circ} \mathrm{C}$ \\
desorb time & $4 \mathrm{~min}$ \\
bake temp & $220^{\circ} \mathrm{C}$ \\
bake time & $8 \mathrm{~min}$
\end{tabular}

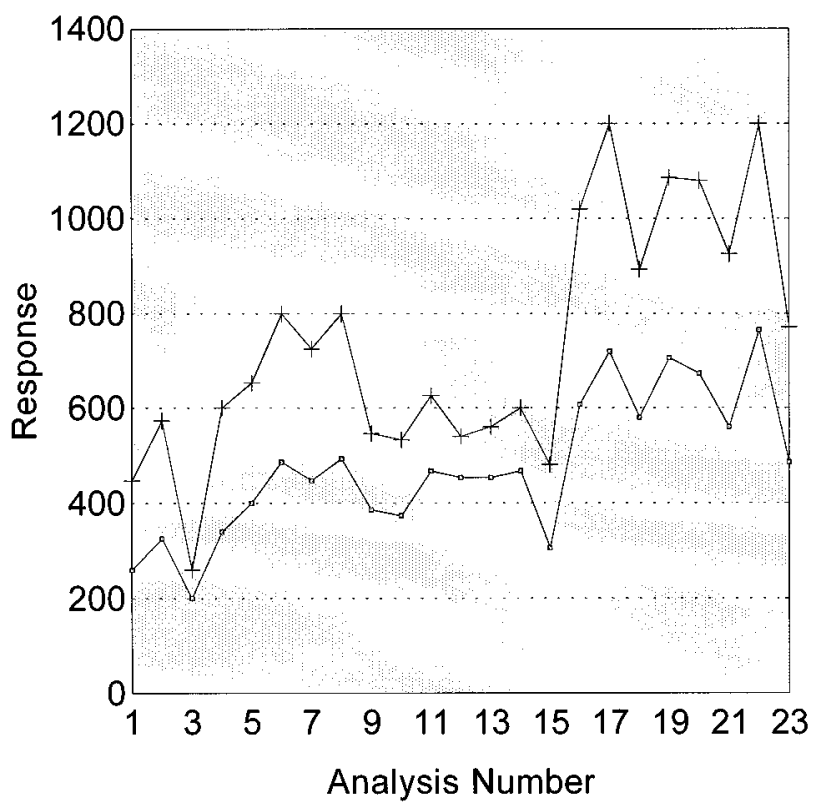

$$
\begin{aligned}
& -\mathrm{BrCl} 2 \mathrm{Me} \\
& + \text { Toluene d-8 }
\end{aligned}
$$

FIGURE 1. Toluene- $d_{8}$ vs bromodichloromethane response (soil B w ).

compounds and thus allowed to interact with the soil for 3 days prior to purging. Byallowing the 3 -day interaction time, any matrix effects affecting target compound extraction will also have had time to affect the extractability of the QRCS. Since the QRCs will be used in the quantitation of the final VOC concentrations, any matrix effects may be accounted for. Thus, the QRCs will more accurately represent the extractability of the target compounds in the soil than the current method's internal standard.

Similar to the current approach in which each target compound is associated with an internal standard, a QRC must also be matched with each target compound. To perform this pairing, instrument response patterns of each target compound and QRC were graphed and visually compared for similar response pattern behavior within each soil type. An exampleof agood responsematch wasidentified for the QRC/target compound pair of toluene- $d_{8}$ and bromodichloromethane in which increases and decreases in instrument responses paralleled each other throughout the sample stream (Figure 1).

Once the target/QRC pairs were identified, a response factor was developed for each PE soil. The response factor calculation is identical to the current method responsefactor except that the QRC is used instead of the internal standard. The response factor is determined as follows:

$$
\mathrm{RF}_{\mathrm{TC}}=\mathrm{A}_{\mathrm{TC}} \mathrm{C}_{\mathrm{QRC}} / \mathrm{A}_{\mathrm{QRC}} \mathrm{C}_{\mathrm{TC}}
$$

where $\mathrm{RF}_{\mathrm{Tc}}$ is the response factor for a given target/ QRC pair,
TABLE 3. Response Ratios Generated by the QRC Approach

\begin{tabular}{lccll} 
& \multicolumn{4}{c}{ horizon } \\
\cline { 2 - 5 } \multicolumn{1}{c}{ target compd/QRCa } & PE C & PE B & PE Bw & NE Ap \\
Bz/Tol- $d_{8}$ & $0.64 / 5.0^{b}$ & $1.02 / 17$ & $0.83 / 8.8$ & $0.52 / 5.2$ \\
TCE/Tol- $d_{8}$ & $0.35 / 4.6$ & $0.28 / 7.3$ & $0.27 / 6.1$ & $0.27 / 4.2$ \\
BrCl $_{2} \mathrm{Me} / T o l-d_{8}$ & $0.61 / 6.1$ & $0.68 / 6.4$ & $0.57 / 7.4$ & $0.67 / 6.4$ \\
PCE/Tol- $d_{8}$ & $0.25 / 4.4$ & $0.14 / 5.3$ & $0.18 / 8.0$ & $0.12 / 11$ \\
EBz/Tol- $d_{8}$ & $* c$ & $*$ & $*$ & $0.19 / 12$ \\
EBz/BFB & $0.69 / 8.4$ & $2.60 / 14$ & $1.93 / 14$ & $*$ \\
1,1,2,2-TTCA/BFB & $2.41 / 5.0$ & $2.03 / 5.5$ & $1.89 / 14$ & $1.56 / 8.2$ \\
Brmfrm/1,1,2,2-TTCA & $0.57 / 2.4$ & $0.73 / 3.2$ & $0.84 / 2.3$ & $0.64 / 2.8$
\end{tabular}

${ }^{a} \mathrm{QRC}$, quantitation reference compound; $\mathrm{PE}$, performance evaluation; NE, Nebraska; $B z$, benzene; Tol- $d_{8}$, toluene- $d_{8}$; TCE, trichloroethylene; $\mathrm{BrCl}_{2} \mathrm{Me}$, bromodichloromethane; $\mathrm{PCE}$, perchloroethylene; $\mathrm{EBz}$, ethylbenzene; BFB, bromofluorobenzene; 1,1,2,2-TTCA, 1,1,2,2-tetrachloroethane; Brmfrm, bromoform. ${ }^{b}$ Second figure is percent relative standard deviation of the eight samples used to generate response ratio. ${ }^{c}$ An asterisk $(*)$ indicates no target compound/QRC pairing identified in this soil.

ATC is thearea of thecharacteristicion of thetarget compound, $A_{\mathrm{ORC}}$ is the area of the characteristic ion of the quantitation reference compound, $\mathrm{C}_{\mathrm{TC}}$ is the concentration of the target compound (ng), and $\mathrm{C}_{\mathrm{QRC}}$ is the concentration of quantitation reference compound (ng). Once the response factors are determined, soil VOC concentrations are calculated asfollows:

$$
\text { concentration }(\mu \mathrm{g} / \mathrm{kg})=\left(\mathrm{A}_{\mathrm{TC}} \mathrm{C}_{\mathrm{QRC}}\right) /\left(\mathrm{A}_{\mathrm{QRC}} \mathrm{RF}_{\mathrm{TC}} \mathrm{W}_{\mathrm{S}}\right)
$$

where $A_{T C}, C_{Q R C}$, and $A_{Q R C}$ are the same as above, $R_{T C}$ is the response factor for the target/QRC pair, and $\mathrm{W}_{\mathrm{S}}$ is the weight of sample (g).

The internal standards in the proposed QRC approach are injected directly at the head of the column by manual injection at the sametime that desorption of target and QRCs from the trap is initiated. By injecting the internal standards at the column head, GC/MS operation can be monitored without any potential interactions with the soil or extraction solution and without being affected by any potential problems with the $P \& T$ system. All internal standards are required to meet an acceptance criterion (e.g., a response window of $\pm 20 \%$ of the peak area of the characteristic ion from the initial calibration) and thus will perform only a QC function and not be used during VOC quantitation.

The first step in the QRC approach is to identify QRC/ target compound pairs through response pattern matching. Toluene- $d_{8}$ was identified as the QRC for benzene, trichloroethylene, bromodichloromethane, perchloroethylene, and ethylbenzene, whilebromofluorobenzene was theQRC match for 1,1,2,2-tetrachloroethane and ethylbenzene in all four spiked soils. Toluene- $d_{8}$ was also identified as a potential QRC for ethylbenzene in the Ap soil only. Three target compounds (bromoform, 1,1-dichloroethane, and 1,1,1trichloroethane) had response patterns that were distinctly different than any of theQRCs, thereby precluding them from quantitation using the proposed approach. However, bromoform response did mimic that of 1,1,2,2-tetrachlorethane fairly well indicating that perhaps 1,1,2,2-tetrachloroethane- 
TABLE 4. Target Compound Recoveries and Precision Estimates by Quantitation Approach

(\% recovery $/ \%$ RSD)

\begin{tabular}{|c|c|c|c|c|c|c|c|c|}
\hline \multirow[b]{2}{*}{ compd } & \multicolumn{2}{|c|}{$\mathrm{PE}^{a} \mathrm{C}$ horizon } & \multicolumn{2}{|c|}{ PE B horizon } & \multicolumn{2}{|c|}{ PE Bw horizon } & \multicolumn{2}{|c|}{ NE Ap horizon } \\
\hline & current & QRC & current & QRC & current & QRC & current & QRC \\
\hline $\begin{array}{l}\mathrm{Bz} \\
\mathrm{TCE} \\
\mathrm{BrCl}_{2} \mathrm{Me} \\
\mathrm{PCE} \\
\mathrm{EBz} \\
\text { Brmfrm } \\
1,1,2,2-\mathrm{TTCA}\end{array}$ & $\begin{array}{r}2 / 30 \\
3 / 24 \\
3 / 25 \\
3 / 24 \\
6 / 17 \\
11 / 20 \\
16 / 16\end{array}$ & $\begin{array}{c}99 / 12 \\
99 / 4 \\
102 / 2 \\
100 / 3 \\
94 / 7 \\
104 / 6 \\
95 / 7\end{array}$ & $\begin{array}{l}18 / 29 \\
17 / 21 \\
21 / 18 \\
10 / 20 \\
25 / 25 \\
14 / 18 \\
16 / 21\end{array}$ & $\begin{array}{c}100 / 15 \\
98 / 10 \\
98 / 3 \\
99 / 7 \\
94 / 7 \\
104 / 5 \\
101 / 7\end{array}$ & $\begin{array}{l}20 / 32 \\
20 / 30 \\
22 / 33 \\
17 / 26 \\
21 / 46 \\
21 / 32 \\
24 / 30\end{array}$ & $\begin{array}{r}105 / 7 \\
101 / 4 \\
98 / 5 \\
100 / 5 \\
103 / 8 \\
95 / 5 \\
93 / 7\end{array}$ & $\begin{array}{l}4 / 23 \\
6 / 28 \\
7 / 27 \\
3 / 20 \\
4 / 23 \\
5 / 25 \\
6 / 25\end{array}$ & $\begin{array}{r}97 / 5 \\
97 / 6 \\
95 / 8 \\
99 / 8 \\
104 / 8 \\
99 / 7 \\
99 / 7\end{array}$ \\
\hline
\end{tabular}

a QRC, quantitation reference compound; PE, performance evaluation; NE, Nebraska; RSD, relative standard deviation; Bz, benzene; TCE, trichloroethylene; $\mathrm{BrCl}_{2} \mathrm{Me}$, bromodichloromethane; PCE, perchloroethylene; $\mathrm{EBz}$, ethylbenzene; 1,1,2,2-TTCA, 1,1,2,2-tetrachloroethane; Brmfrm, bromoform.

$\mathrm{d}_{8}$ might bea possibleQRC for bromoform. Further research to identify QRCs for these compounds is warranted.

Once the QRC/target compound pairs were identified, response factors were calculated. The first eight analyses for each spiked soil were used to generate an average response factor for the QRC/target pairs. Even though response patterns were similar within a given horizon, the calculated response ratios differed among soils (Table 3 ). Differing response ratios for the same $\mathrm{QRC/target} \mathrm{compound} \mathrm{pairs}$ amongthe spiked soils suggest theexistence of a matrix effect.

Target VOC concentrations were calculated using either the RF generated from the internal standards (i.e., the current method) or from theQRC (i.e., the QRC approach). Theresults were then compared to the $100 \mu \mathrm{g} / \mathrm{kg}$ concentration of the target compound spike. VOC recoveries using the current method resulted in recoveries ranging from $2-27 \%$ of the known VOC concentrations in all four soils (Table 4). In contrast, when VOC concentrations were determined using the QRC approach, relative recoveries ranged from 93-105\% of the spiked VOC concentrations in all four soils.

The improved accuracy of the QRC approach is attributed to the ability of the QRC to better represent the reactions occurring among the soil, VOCs, and headspace than the internal standard currently used in VOC quantitation. Since the QRCs were injected into the sample during initial VOC spiking and allowed time to interact with the soil matrix, any losses occurring due to microbial degradation, abiotic degradation, micropore sorption, or volatilization to the vial headspace presumably occurred to the QRCs and target compounds in the same relative proportions. Thus, even though VOCs may have been lost in unknown quantities, the final target VOC concentrations can beadjusted back to their initial concentrations based on equivalent losses to the QRC and quantitation using QRC-generated RFs. This idea is supported by the results from the $C$ horizon PE samples in which one batch was stored for 2 days and the other for 3 days. In this case, the instrument response of the samples stored for 2 days was noticeably greater than those stored for 3 days, indicating that someVOC loss had occurred. However, the final VOC relative recoveries were nearly the same and had low percent relative standard deviations (i.e., good precision) ranging from only $2-15 \%$ (Table 4 ) among the replicates.

Precision values ranged from 16 to $46 \%$ relative standard deviation (RSD) using the current approach as compared to 2-15\% RSD by the QRC approach (Table 4). While these relativedifferences may seem large, the differences in absolute concentrations between each calculation method are similar (i.e., low target compound recoveries using the current method result in high RSD for small variations in absolute recoveries). These results indicate that both methods are equally precise on an absolute basis but due to the more accurate percent recoveries obtained by the QRC approach, the QRC approach appears to be more precise.
TABLE 5. Average Target Compound Recoveries from Contact Time Studya

\begin{tabular}{lcc} 
& \multicolumn{2}{c}{ (\% recovery/\% RSD) } \\
\cline { 2 - 3 } \multicolumn{1}{c}{ compd $^{b}$} & current method & QRC approach \\
$\mathrm{Bz}$ & $2.3 / 21$ & $97 / 8.2$ \\
$\mathrm{TCE}$ & $3.8 / 21$ & $95 / 8.2$ \\
$\mathrm{BrCl}{ }_{2} \mathrm{Me}$ & $5.1 / 19$ & $98 / 4.9$ \\
$\mathrm{PCE}$ & $1.8 / 23$ & $93 / 6.0$ \\
$\mathrm{EBz}$ & $2.2 / 18$ & $99 / 5.7$ \\
$\mathrm{Brmfrm}$ & $3.5 / 17$ & $97 / 2.6$ \\
$1,1,2,2-\mathrm{TTCA}$ & $4.0 / 15$ & $99 / 7.2$
\end{tabular}

${ }^{a}$ This study was performed on the spiked Nebraska Ap horizon only. ${ }^{b} \mathrm{RSD}$, relative standard deviation; $\mathrm{Bz}$, benzene; TCE, trichloroethylene; $\mathrm{BrCl}_{2} \mathrm{Me}$, bromodichloromethane; $\mathrm{PCE}$, perchloroethylene; $\mathrm{EBz}$, ethylbenzene; 1,1,2,2-TTCA, 1,1,2,2-tetrachloroethane; Brmfrm, bromoform.

To further demonstrate the advantage of using the QRC approach, comparisons were made of target compound recoveries among the three different $P E$ soils. Higher target VOC recoveries werefound in the $B$ and $B$ w than the $C$ horizon PE samples using the current quantitation method (Table 4). A 10 - and 30-fold increase in total organic carbon (TOC) content (Table 1) in the B and Bw horizonsfrom the C horizon, respectively, may account for this result since TOC has been shown to be a significant factor in VOC retention (24-27). In contrast, by adding QRCs at the time of initial VOC spiking and thusallowing time for matrix interaction, thesamelevels of relative recoveries were calculated for all three spiked PE soils, regardless of TOC content and other potential matrix effects. Therefore, by using the QRC approach, comparisons in VOC concentrations between soils and sites can be made based on actual differences in VOC concentration with minimal concern about matrix effects caused by differing TOC contents or particle-size distributions.

The influence of contact time between the QRC and soil (as determined after $3 \mathrm{~h}, 2$ days, and 3 days) appeared to be minor in the spiked Ap soil (Table 5). Average target compound relativerecoveries ranged from 93 to $99 \%$ (absolute recoveries ranged from 88 to $105 \%$ ) with RSDs ranging from 2.6 to $8.2 \%$ using the QRC approach. In contrast, average recoveriesusing thecurrent quantitation method ranged from 1.8 to $5.1 \%$ of the known concentration. Theseresults indicate that the QRC approach can accurately monitor VOC concentrations regardless of holding times, soil matrix effects, compound class, and VOC losses to the system through time and sample handling, preparation, and analysis. Therefore, even if the samples are spiked with the QRCsin the laboratory upon sample receipt, the QRC approach is useful in monitoring VOC losses during future sample processing steps. However, it is recommended that the samples be spiked at the earliest time, preferably in the field, to allow for full soil/ QRC interaction and VOC loss monitoring. 
Internal standards, introduced to the head of the GC column by manual injection, provided response variations of $\pm 20 \%$ indicating consistent, precise GC/MS operation. Direct injection of the internal standards in the QRC approach is designed to monitor just the GC/MSfunction and to totally eliminate any potential extraction solution/soil matrix interaction with the internal standard as required by definition (18). Auto-injection of internal standards should reduce variation and allow for even tighter recovery windows, resulting in an continuous, reliable monitor of the GC/MS.

\section{Acknowledgments}

The U.S. Environmental Protection Agency (EPA), through its Office of Research and Development (ORD), funded and collaborated in the extramural research described here. It has been subjected to the Agency's peer review and has been approved as an EPA publication. Mention of trade names or commercial products does not constitute endorsement or recommendation for use.

\section{Literature Cited}

(1) Siegrist, R. L.; Jenssen, P. Environ. Sci. Technol. 1990, 24, 13871392.

(2) Hewitt, A. D. Proceedings, 16th Annual Army Environmental R\&D Symposium; Williamsburg, VA, 1992.

(3) Hewitt, A. D. Losses in trichloroethylenefrom soil during sample collection, storage and laboratory handling; U.S. Army Cold Regions Research and Engineering Lab.: Hanover, NH; 1994; SR94-8.

(4) Urban, M. J.; Smith, J. S.; Schultz, E. K.; Dickinson, R. K. Abstracts of Papers, Proceedings Waste Testing and Quality Assurance; U.S. Environmental Protection Agency: Washington, DC, 1989.

(5) Hewitt, A. D.; Jenkins, T. F.; Grant, C. L. Am. Environ. Lab 1995, 7. 25-28.

(6) Jenkins, R. A.; Bayne, C. K.; Maskarinec, M. P.; Johnson, L. H.; Holladay, S. K.; Tomkins, B. A. Experimental determination of pre-analytical holdingtimesfor volatileorganicsin selected soils; Abstract from National Symposium on Measuring and Interpreting VOCs in Soils: State of the Art and Research Needs; U.S. Environmental Protection Agency: Las Vegas, NV, 1993.

(7) King, P. H. Evaluation of sampleholding times and preservation methodsfor gasol inein fine-grained sand; Abstract from National Symposium on Measuring and Interpreting VOCs in Soils: State of the Art and Research Needs; U.S. Environmental Protection Agency: Las Vegas, NV, 1993

(8) Anderson, T. A.; Beauchamp, J. J.; Walton, B. T. J. Environ. Qual. 1991, 20, 420-424.
(9) Dragun, J. 1988. The Soil Chemistry of Hazardous Materials; Hazardous Materials Control Research Institute: Silver Springs, MD, 1988; $458 \mathrm{pp}$.

(10) Dilling, W. L.; Tefertiller, M. B.; Kallos, G.J. Environ. Sci. Technol. 1975, 9, 833-838.

(11) Steinberg, S. M.; Pignatello, J. J.; Sawhney, B. L. Environ. Sci. Technol. 1987, 21, 1201-1208.

(12) Maskarinec, M. P.; Johnson, L. H.; Holladay, S. K. Recommendations for holding times of environmental samples; Proceedings Waste Testing and Quality Assurance; U.S. Environmental Protection Agency: Washington, DC, 1988.

(13) Amin, T. A.; Narang, R. S. Anal. Chem. 1985, 57, 648-651.

(14) Sawhney, B. L., Pignatello, J. J., Steinberg, S. M. J. Environ. Qual. 1988, 17, 149-152.

(15) Pignatello, J. J. Environ. Toxicol. Chem. 1990, 9, 1107-1115.

(16) Pavlostathis, S. G.;Jaglal, K. Environ. Sci. Technol. 1991, 25, 274 279.

(17) Minnich, M. Behavior and determination of volatile organic compounds in soil: A literature review; U.S. Environmental Protection Agency: Las Vegas, NV, 1993; EPA/600/R-93/ 140.

(18) American Public Health Association. Standard Methods for the Examination of Water and Wastewaters, 18th ed.; American Public Health Assoc.: Washington, DC, 1992.

(19) U.S. Environmental Protection Agency. Test Methods for Evaluating Solid Waste, SW-846, 3rd ed; Office of Solid Waste and Emergency Response, U.S. Environmental Protection Agency: Washington, DC, 1986.

(20) Charles, M. J.; Simmons, M. S. Anal. Chem. 1987, 59, 12171221.

(21) Olynyk, P. W.; Budde, W.; Eichelberger, J. J. Chromatogr. Sci. 1981, 19, 377-382.

(22) U.S. Environmental Protection Agency. Performance Tests for the Evaluation of Computerized Gas Chromatography/Mass Spectrometry Equipment and Laboratories; U.S. Environmental Protection Agency: Washington, DC, 1980; EPA/600/4-80/025.

(23) Gee, G. W.; Bauder, J. W. Particle Size Analysis. In Methods of Soil Analysis; Klute, A., Ed.; Agronomy 9; American Society of Agronony: 1986; pp 383-411.

(24) Brusseau, M. Environ. Sci. Technol. 1991, 25, 1747-1752.

(25) Chiou, C. T.; Kile, D.; Malcolm, R. Environ. Sci. Technol. 1988, $22,298-303$.

(26) Southworth, G.; Keller, J. Water, Air Soil Pollut. 1986, 28, 239248.

(27) Wu, S.; Gschwend, P. Environ. Sci. Technol. 1986, 20, 717-725.

Received for review October 9, 1996. Revised manuscript received March 6, 1997. Accepted March 6, 1997. ${ }^{\circledR}$

\section{ES960867Q}

${ }^{\otimes}$ Abstract published in Advance ACS Abstracts, May 15, 1997. 